\title{
Observations of synchronized spawning, larval survival and settlement in corals of the genus Acropora in Lakshadweep, India
}

\author{
S. Nina Tabitha ${ }^{1,2}$, Kevin Shimrone Moses $^{1}$ and Rajkumar Rajan ${ }^{1, *}$ \\ ${ }^{1}$ Zoological Survey of India, Marine Regional Centre, Chennai 600 028, India \\ ${ }^{2}$ University of Madras, Chennai 600 005, India
}

\begin{abstract}
Reports of synchronous spawning in corals of Lakshadweep reefs, India have been purely anecdotal. To understand factors that underlie coral spawning in Lakshadweep, we studied four Acropora species ( $A$. muricata, $A$. cytherea, $A$. hemprichii and $A$. nobilis) in Kavaratti atoll from February to March 2018. These species were observed in situ for oocyte maturation, and the larvae of species $A$. muricata and $A$. cytherea were assessed for survival and settlement rates. Observations on oocyte maturation indicated possible spawning closer to the immediate full moon of species in which $7-28 \%$ of the colonies had mature oocytes. Thus, A. muricata, A. cytherea and A. hemprichii spawned on 25 and 26 February 2018, demonstrating multi-specific synchronous spawning, whereas $A$. nobilis spawned on 21 March 2018. Larval survival rates revealed a steep die-off beginning on day 12 postspawning and maximum survival up to 24-27 days. Settlement occurred between 12 and 20 days for $A$. muricata and between 12 and 22 days for $A$. cytherea. The comparatively low survival duration and fewer larvae surviving for settlement show that maximum settlement could be heavily compromised. However, the study reveals that the comparatively late onset of settlement (from day-12, post-spawning) and the broader settlement window of 8-10 days could allow room for long-distance dispersal of larvae. Further studies on this front are required to gather a better picture.
\end{abstract}

Keywords: Acropora genus, coral reproduction, larvae survival, oocyte maturation, synchronized spawning.

SYNCHRONIZED spawning has been observed in corals during sexual reproduction, which occurs either through broadcasting or brooding ${ }^{1,2}$. Broadcasting is the process where coral polyps release bundles of sperm and eggs into the water column, and thus fertilization is external; brooders release hatched planula developed from internally fertilized eggs. Brooding and broadcasting gametes are found in both gonochoristic or hermaphroditic corals ${ }^{2}$. Broadcast and synchronized spawning in corals are vital

*For correspondence. (e-mail: raj@zsi.gov.in) to accomplishing high fertilization rates, and increasing the probability of genetic mixing between colonies ${ }^{3,4}$. Spawning also indicates the presence of adult colonies capable of maintaining sustainable and self-seeding populations on a reef ${ }^{5}$. Most scleractinian corals are simultaneous broadcasting hermaphrodites that spawn annually, usually during the warmest months in the year, although the exact duration differs from location to location based on latitudinal and temperature variations ${ }^{4,6-11}$. Since spawning is influenced on a regional scale, depending on factors like time of the year, lunar phase, seawater temperature and availability of settlement cues, it is necessary to make observations on a regional scale ${ }^{3,7,12}$. Besides, it is also critical to assess survival and settlement rates of coral larvae to understand spawning success.

We undertook the present study given the lack of observations of coral spawning in Lakshadweep reefs, and the importance of such knowledge to the conservation of coral species. We observed four species of the coral genus Acropora, viz. A. muricata, A. hemprichii, A. cytherea and $A$. nobilis, for their spawning patterns. Experiments to study rates of survival of larvae were executed for two species, A. muricata and A. cytherea, the results of which are presented here.

\section{Methods}

\section{Preliminary observations}

Kavaratti atoll $\left(10^{\circ} 34^{\prime} 22.25^{\prime \prime} \mathrm{N} ; 072^{\circ} 37^{\prime} 49.15^{\prime \prime} \mathrm{E}\right)$ in the Lakshadweep Islands was selected as the study site. The atoll, elliptical in shape, is aligned in a northeast direction and has a broader lagoon to the west, and the island to the east (Figure 1). The total extent of reef is about 3.3 sq. $\mathrm{km}$ (ref. 13). Although the live coral cover estimated at the reef slopes was only 5-9.2\%, thickets of Acropora spp. of $\sim 76 \%$ cover were found in the lagoon near the reef crest. We inferred the approximate time of coral spawning for the Lakshadweep atolls from spawning occurrences reported from neighbouring reef regions and reports of spawn slick for the study site reported from Agatti atoll, Lakshadweep ${ }^{8,14,15}$. We carried out preliminary observations between January and March 2017, 


\section{RESEARCH ARTICLES}

observing oocyte maturation in randomly selected colonies. Colonies with pigmented oocytes were scored as 'reproductively mature', whereas those with unpigmented oocytes and empty mesenteries were scored as 'reproductively immature' as these were considered to have already spawned or highly unlikely to spawn within the next lu-

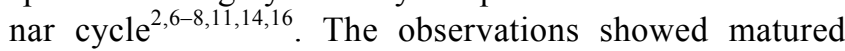
oocytes from 11 February 2017 in A. muricata, and the spawn slicks on 7 March 2017. Taking these as inference, we planned the study to record spawning in selected Acropora species the following year (2018).

\section{Observation of maturation in oocytes}

A sampling station about 500 sq. $m$ was marked $\left(10^{\circ} 34^{\prime} 22.25^{\prime \prime} \mathrm{N} ; 072^{\circ} 37^{\prime} 49.15^{\prime \prime} \mathrm{E}\right)$ in the lagoon, which suited the criteria of having the highest abundance and area cover of Acropora spp. (Figure 1). We chose A. muricata, A. cytherea, A. hemprichii and A. nobilis as candidate species given their sufficient colonies for sam-

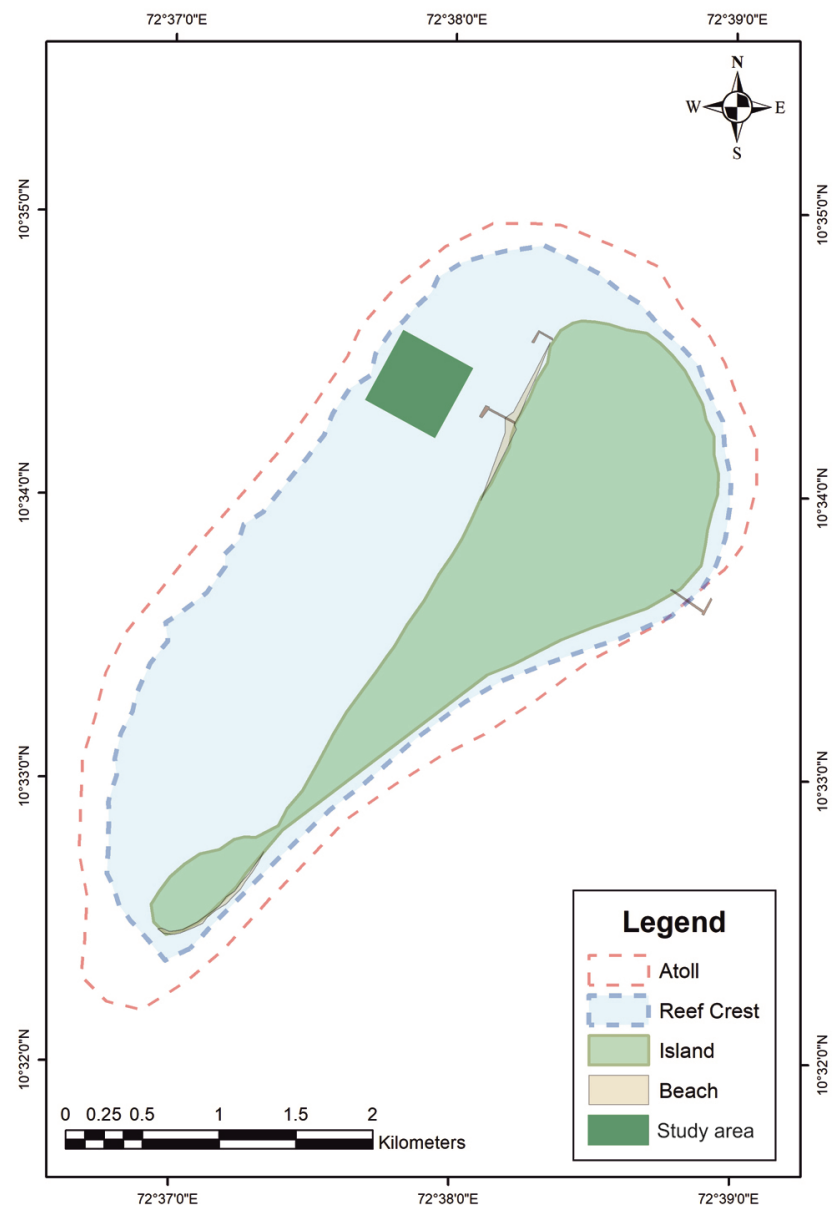

Figure 1. The study site in Lakshadweep, India (marked here by a darker green shade) was selected as it had an abundance cover of the targeted Acropora spp. The site has an extent of $500 \times 500$ sq. m, and was located on the northwestern side of the Kavaratti atoll inside the lagoon $\left(10^{\circ} 34^{\prime} 22.25^{\prime \prime} \mathrm{N} ; 072^{\circ} 37^{\prime} 49.15^{\prime \prime} \mathrm{E}\right)$. pling within 500 sq. $\mathrm{m}$ of the marked study site. The colonies were sampled from 11 February 2018 onwards the date decided based on observation of mature oocytes in A. muricata colonies in the preliminary observations during January-March 2017. Fifteen random colonies of each species with $>30 \mathrm{~cm}$ of colony diameter were sampled to observe the maturation of oocytes ${ }^{14}$. One or two fragments were broken from each colony, 5-15 cm away from the tip of the sterile axial corallite ${ }^{14,17}$. The oocyte maturation was scored as noted above.

\section{Collection of spawn}

Spawn traps were used for the collection of egg-sperm bundles. The traps were designed to be conical in shape (made using $100 \mu \mathrm{m}$ plankton nets that measured $147 \mathrm{~cm}$ in height and had a square base that covered an area of $90 \mathrm{~cm}^{2}$ ) with the tapered end opening into a sleeve provided with a drawstring to attach a plastic collection container of 1 litre volume (Figure 2). The base was anchored using small weights in such a way to cover the colony, and the sleeve of the net was fitted with floats

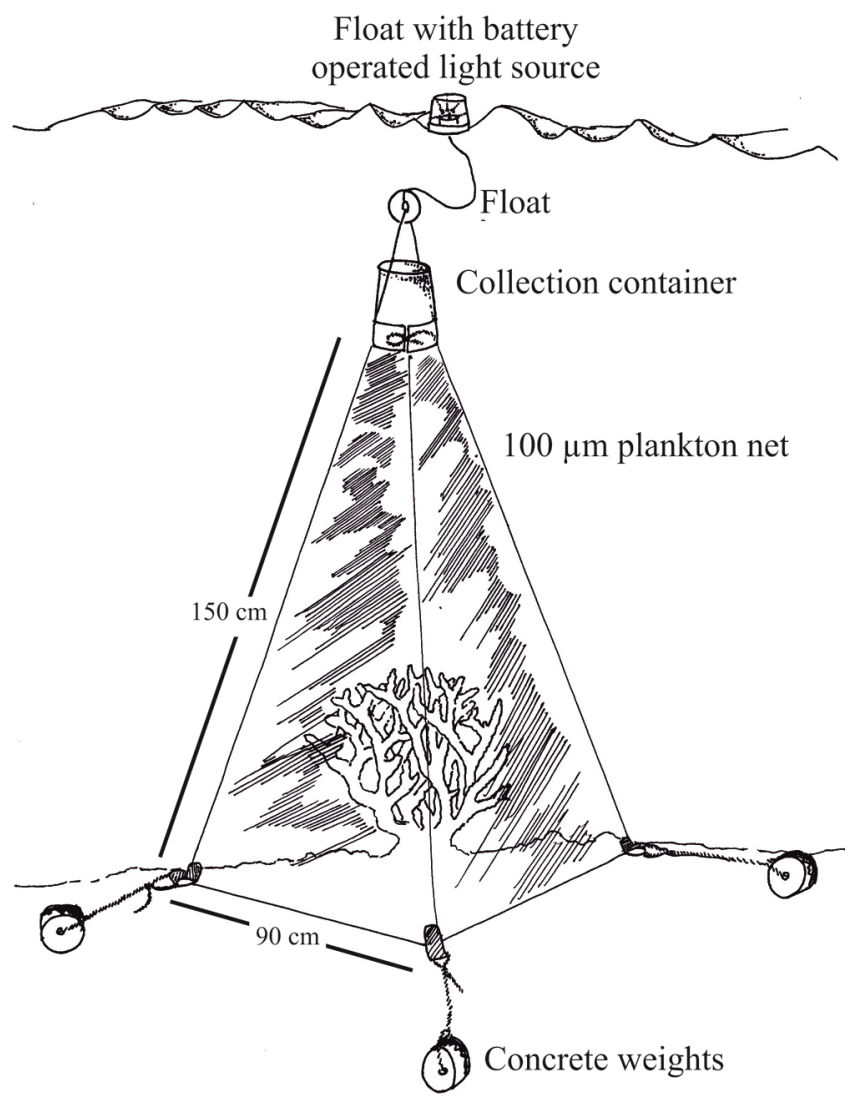

Figure 2. Diagrammatic representation of the spawn-trap fabricated to collect coral spawn. It consists of weights made of concrete anchors, and $100 \mu \mathrm{m}$ plankton net stitched conically and tapering towards the tail, where a collection chamber could be easily attached using a draw string. The collection chamber is attached to a float to make it stand perpendicular. 
(in order to gather and collect the buoyant spawn) that maintained the conical shape of the net and kept the collection container upright. Two gravid colonies for each of the individual species were set up with nets every evening at $1730 \mathrm{~h}$. The nets were removed the next morning at $0630 \mathrm{~h}$. Any spawning activity was monitored by keeping overnight vigils in a boat, and the collection containers checked for spawn every $30 \mathrm{~min}$ throughout the period of deployment ${ }^{14}$. This operation was followed from 11 February 2018 until the spawning night. Observers either snorkelled or SCUBA-dived during deployment and monitoring. Once spawning was complete, the containers possessing spawn were carefully removed to be transported to the laboratory within $2 \mathrm{~h}$ for effecting fertilization. The event of spawning was videographed.

\section{Fertilizing the eggs}

Spawns from different colonies of each species were transferred to circular polyvinyl culture tanks, maintained at a sheltered enclosure containing 100 litres of filtered seawater, stirred gently to aid fertilization, and left undisturbed for $30 \mathrm{~min}$ to avoid polyspermy ${ }^{14}$. Thereafter the tanks were topped up to 200 litres and maintained. The water was replaced periodically (once in every $6 \mathrm{~h}$ on the first two days, and every $24 \mathrm{~h}$, for the following 10 days $^{16}$, and every $48 \mathrm{~h}$ thereafter) by siphoning out approximately half the volume of water through a $60-\mu \mathrm{m}$ filter cloth and replacing it with fresh filtered seawater to cope with rising nitrate levels due to decaying unfertilized eggs. Lipid accumulation along the sides of the tank due to dead larvae and unfertilized eggs was removed by wiping with a sponge and skimming the surface foam whenever observed. The water was aerated continually, and the water quality tested daily using a reef-aquarium water quality test kit (API marine reef master test kit). The temperature of water was maintained between $28^{\circ} \mathrm{C}$ and $30^{\circ} \mathrm{C}$ by providing proper shading ${ }^{17}$. The approximate stocking density of planula larvae obtained was 372 and 328 larvae/1 for $A$. muricata and $A$. cytherea respectively. Only a few eggs/larvae were obtained from $A$. nobilis and $A$. hemprichii were therefore not considered for the experiments to assess larval survival and settlement rates.

\section{Estimation of larval survival rates}

The survival rates were estimated for the larvae of A. cytheria and A. muricata. The number of larvae surviving in the culture throughout the duration (28 days) was enumerated from $50 \mathrm{ml}$ aliquots $(n=5)$ taken every $48 \mathrm{~h}$ from the culture tanks. Care was taken to ensure a homogenous distribution of larvae in the water column by gently stirring the culture using a spatula during sampling. It may be noted that the larvae in the culture tanks did not settle throughout the study duration, for which inducement by introducing crustose coralline algae was required $^{18-21}$.

\section{Estimation of larval settlement rates}

The experiments for larval settlement rates were carried out from the sixth day of culture (day 2 of the larval life), when the larvae of $A$. muricata and $A$. cytherea, obtained fully formed pear shapes ${ }^{17}$. Ten randomly sampled individuals were introduced into shallow polyethene cups $(n=3)$ containing $10 \mathrm{ml}$ of filtered seawater with $10 \mathrm{mg}$ of crustose coralline algae (CCA) slurry to induce them

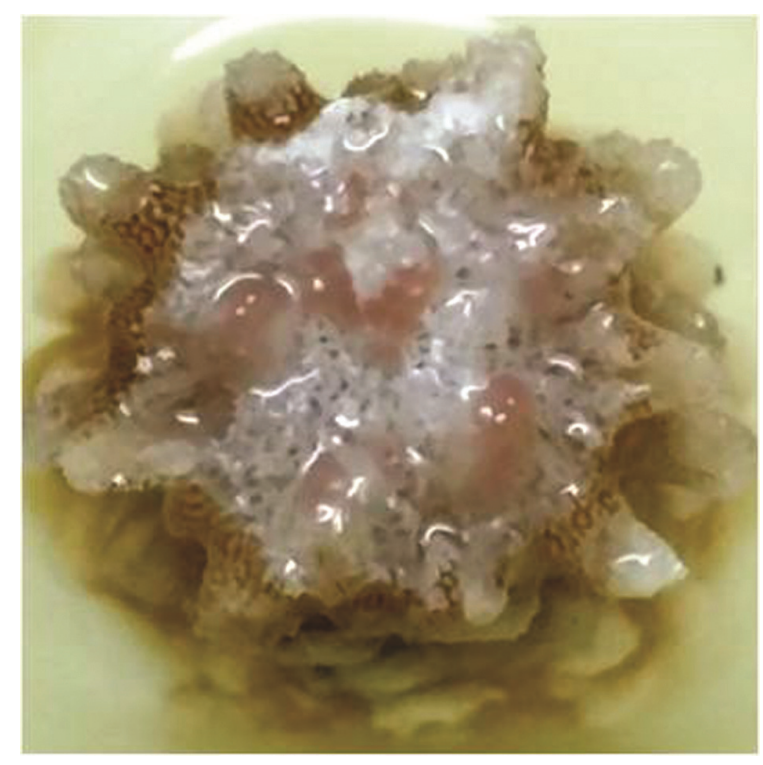

Figure 3. A broken fragment of Acropora muricata showing pigmented oocytes in the mesenteries, sampled on 20 February 2018. An estimate of the percentage of colonies with mature oocytes was arrived at by breaking a coral fragment to note the presence or absence of pigmented oocytes.

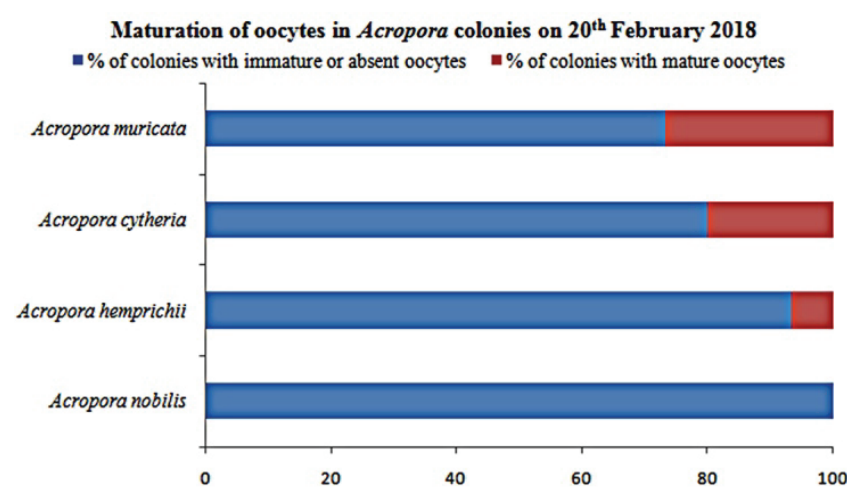

Figure 4. Percentage of colonies with mature oocytes as of 20 February 2018 , in Acropora muricata (27\%), Acropora cytherea $(20 \%)$ and Acropora hemprichii (7\%). Acropora nobilis colonies were not observed with pigmented oocytes, indicating a later spawning date for this species. 


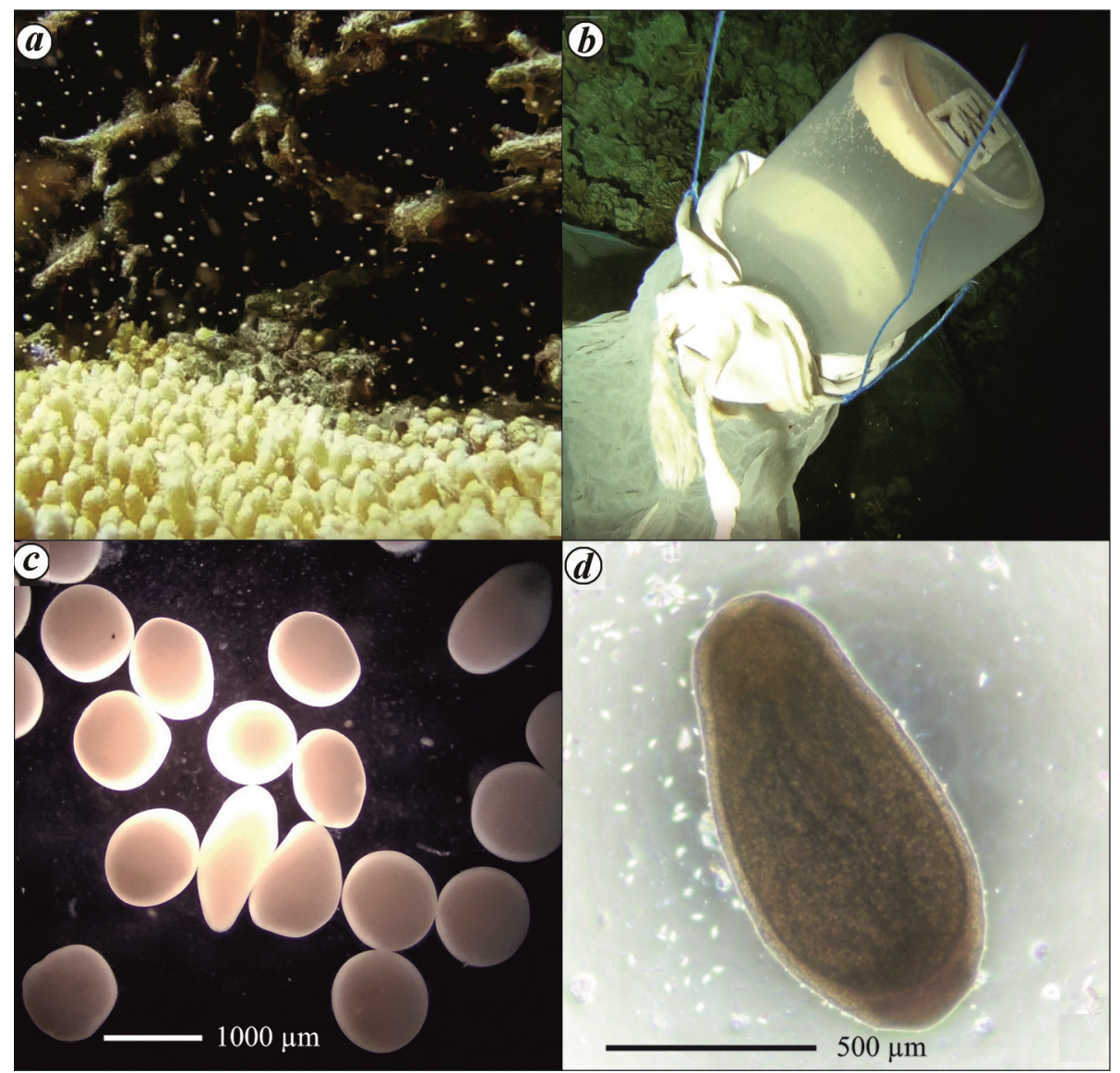

Figure 5. Documentation of spawning in A. cytherea: a, A colony releasing spawn in situ; $\boldsymbol{b}$, Sperm and egg bundles trapped in the collection chamber of the spawn-trap; $\boldsymbol{c}$, Fertilized eggs observed under a microscope; $\boldsymbol{d}$, A fully developed planula larvae.

to settle ${ }^{18-21}$. The number of larvae settled and freefloating was counted after $48 \mathrm{~h}$ (on day 8 of the culture) under a dissection microscope following Pollock et al. ${ }^{22}$. The experiment due for observation on day 10 was begun immediately after completing the first observation, i.e. on day 8 , following the same protocol. The control $(n=3)$ consisted of the same set-up without the introduction of CCA. The experiment was repeated ten times for 18 days until day 26 of culture.

\section{Results}

\section{Maturation of oocytes}

Three of the four species of Acropora studied were observed with mature pigmented oocytes, beginning 11 February 2018 (Figure 3). Figure 4 shows the score as on 20 February 2018 for each species $(n=15)$. A. muricata, $A$. cytherea and $A$. hemprichii exhibited pigmented oocytes at the rate of $7-28 \%$, indicating possible spawning of these species within the next full moon, as deduced from earlier studies ${ }^{1,2,6-8,11}$, falling on 2 March 2018. A. nobilis, on the other hand, did not exhibit pigmented oocytes at this time, but was observed with mature oocytes the following month.

\section{Occurrence of spawning}

We reported synchronous spawning during 25-26 February 2018 for the three species of Acropora, which were predicted to spawn closer to the full moon night on 2 March 2018 (Table 1; Figure 5a). A. cytheria and $A$. hemprichii spawned at $2130 \mathrm{~h}$ on 25 February 2018. However, out of two colonies per species laid with spawn-traps, spawn (egg-sperm bundles) could be observed on one each, in these species (Figure $5 b$ ). All three species (including $A$. muricata) spawned on the next day (26 February 2018), four days before full moon, and the egg-sperm bundles were collected on all the six (two each for three species) traps placed. Spawn-slick was observed across the lagoon in the night and along the southwestern shores of the island on the next morning (Figure 6). Observation of coral fragments of the species spawned showed empty mesenteries until the first week of April 2018, confirming the absence of split spawning pattern in these species (Figure 7). A. nobilis spawned on 
Table 1. Species studied, date and time of spawning, particulars of spawn-traps, temperature and moon-visibility data at the time of spawning

\begin{tabular}{llcccc}
\hline \multirow{2}{*}{ Species } & Date of spawning & Time & $\begin{array}{c}\text { Number of traps laid } \\
\text { (two for each species) }\end{array}$ & $\begin{array}{c}\text { Temperature } \\
\left({ }^{\circ} \mathrm{C}\right)\end{array}$ & $\begin{array}{c}\text { Moon visibility } \\
(\%)\end{array}$ \\
\hline Acropora cytherea & 25 February 2018 & $9.00-10.00 \mathrm{pm}$ & One full, one empty & 31 & 69 \\
& 26 February 2018 & $9.30-10.00 \mathrm{pm}$ & Two full & 30 & 79 \\
Acropora hemprichii & 25 February 2018 & $9.00-10.00 \mathrm{pm}$ & One full, one empty & 31 & 30 \\
& 26 February 2018 & $9.30-10.00 \mathrm{pm}$ & Two full & 30 & 79 \\
Acropora muricata & 26 February 2018 & $9.30-10.00 \mathrm{pm}$ & Two full & 31 & 79 \\
Acropora nobilis & 21 March 2018 & $9.30-10.00 \mathrm{pm}$ & Two full & & 18 \\
\hline
\end{tabular}

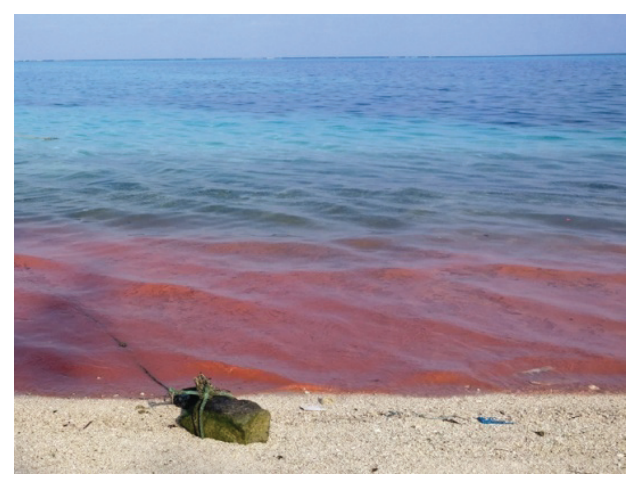

Figure 6. Spawn slick observed on 27 February 2018 at 8:30 am on the southwestern shores of Kavaratti Island, Lakshadweep.

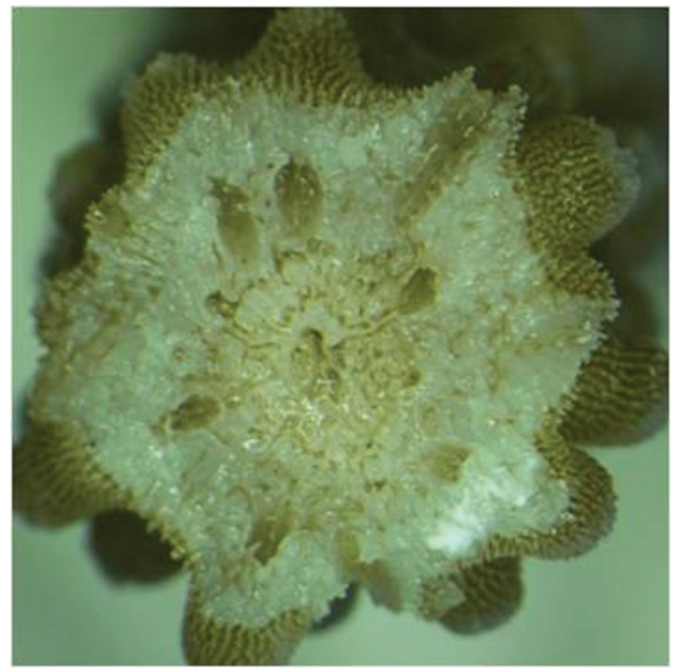

Figure 7. A broken coral fragment sampled after the spawning event on 28 February 2018. The fragments had mesenteries empty of oocytes.

21 March 2018. However, oocyte maturation rates in this species could not determined due to logistic constraints.

\section{Larvae survival experiment}

The larvae were noticed in the water column on the fourth day after effecting fertilization (Figure $5 d$ ). The number of eggs that developed into planula larvae was highest on day 6 of the culture for $A$. cytherea (average 17.8 larvae $/ 50 \mathrm{ml}, n=5)$, and day 10 for $A$. muricata

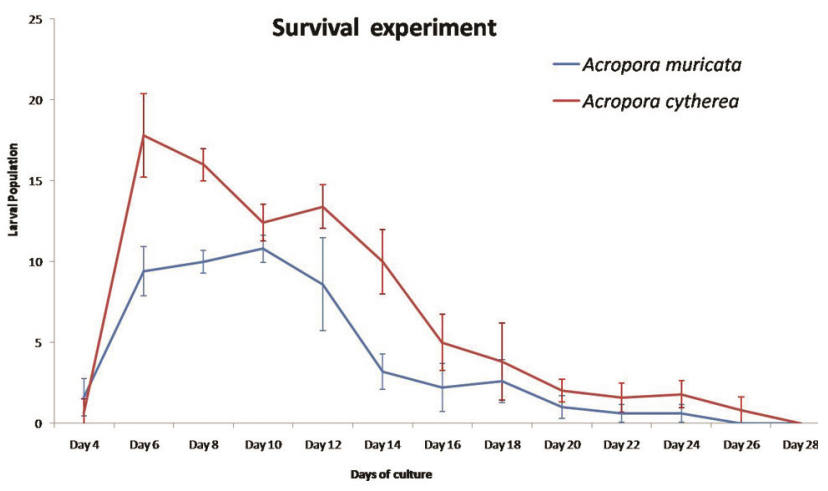

Figure 8. The larval population of $A$. cytherea in the culture, estimated by taking $50 \mathrm{ml}$ aliquots $(n=5)$ at an interval of $48 \mathrm{~h}$. Both $A$. muricata and $A$. cytherea exhibited similar survival patterns. The peak in larval numbers was observed between days 6 and 10. The decline in numbers was observed from day 12 and until day 16, indicating heavy die-off. A gradual decline was observed thereafter, with the larvae surviving for a maximum of 27 days.

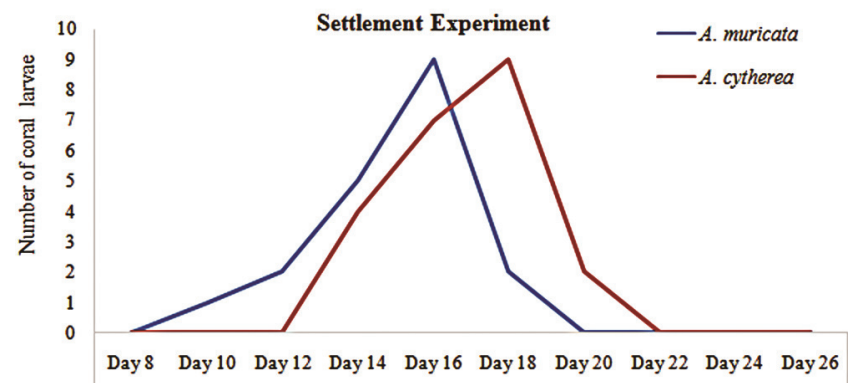

Figure 9. Experiments to estimate the larval settlement rates were carried out from day 6, after the larvae had formed pear shapes. Ten randomly sampled individuals were introduced into shallow polyethene cups $(n=3)$ containing $10 \mathrm{ml}$ of filtered seawater with $10 \mathrm{mg}$ of crustose coralline algae (CCA) slurry to induce them to settle. The number of larvae settled at the end of $48 \mathrm{~h}$ was counted. The experiment was repeated with larvae on day 8,10 and in that order until day 26 . The graph shows the number of larvae observed as settled after every $48 \mathrm{~h}$. The larvae in the control containers (without CCA), did not settle throughout the experiment.

(average 9.4 larvae $/ 50 \mathrm{ml}, n=5$ ). The two species showed similar survival patterns (a one-way ANOVA performed showed $P$ values $=0.22$, alpha value $=0.05$ ), with a steep decrease in larval numbers after the 12th day of culture, followed by a gradual decline (Figure 8). The 


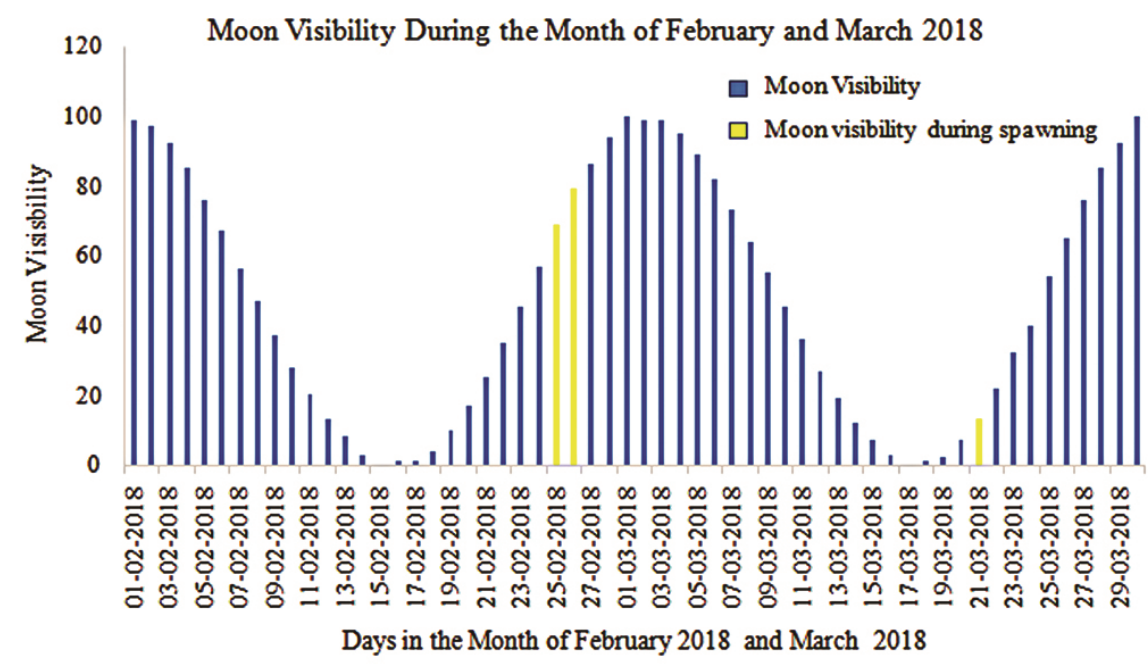

Figure 10. Synchronous spawning was observed in A. cytherea and A. hemprichii respectively, on the fourth and fifth day before the full moon, while A. nobilis spawned three days after new moon.

larvae of $A$. muricata and $A$. cytherea survived up to days 25 and 27 respectively.

\section{Larval settlement experiment}

The larvae of $A$. muricata settled between 12 and 20 days and those of $A$. cytherea between 12 and 22 days. The peak larval settlement was on day 16 for $A$. muricata and day 18 for $A$. cytherea (Figure 9). Both the species followed a similar pattern of settlement. None of the larvae in the control setups settled, proving that CCA is essential to induce settlement ${ }^{22}$.

\section{Discussion}

Until 1970 scientists did not realize that certain coral species were capable of fertilization externally, apart from the general assumption that corals reproduced internally by brooding larvae ${ }^{6}$. This discovery led to further research on the sexual reproduction of corals that reproduced externally, also known as broadcast spawners. The findings that corals which broadcast egg-sperm bundles could have long dispersal ranges and multi-specific synchronous spawning enables genetic mixing underscore the importance of broadcast spawning to the well-being of reefs ${ }^{3-6,17}$, and hence the need for studies to understand spawning patterns of coral species in reefs.

There is little information on coral spawning from Indian reefs, and studies on behaviour of coral larvae are unavailable. Previous reports in Indian reefs show spawning occurring in $\mathrm{March}^{23}$, except for a report from the Andaman Islands, where it is in February ${ }^{24}$. For Lakshadweep reefs, a previous report of spawn-slick was on 18 March 2013 (ref. 15). The review of coral spawning in the Arabian Sea and western Indian Ocean by Howells et al. ${ }^{8}$ in 2014, which also covers previous spawning reports from Lakshadweep and the Maldives, points to the general occurrence in March.

We observed pigmented oocytes in February (11 February 2018 onwards) in three of the Acropora species (A. muricata, A. hemprichii and A. cytheria) with 7-28\% of the colonies exhibiting pigmented oocytes on 20 February 2018 (Figure 3 ). These species were predicted to spawn during or closer to the approaching full moon and spawned five days prior to the full moon on 25-26 February. A. nobilis, which did not exhibit pigmented oocytes in February, spawned on 21 March 2018, i.e. five days after new moon. The moon visibility graph shows the two spawn timings of the species observed in Lakshadweep (Figure 10).

Coral spawning before full moon is a rare occurrence $^{25-27}$; most reported spawning occurrences were 4-7 nights after full moon across the Great Barrier reef, Taiwan, Japan, Red Sea, Hawaii and Florida ${ }^{2,12}$. However, as observed by Hayashibara et al. $^{27}$, during 1989-91 in Akajima, Japan, and by Babcock et al. ${ }^{2}$ in the Great Barrier Reef, Australia in 1985, where the lunar phases of mass spawning were not consistent, the peak temperature could trigger spawning before a full moon ${ }^{25}$. The annual rise in sea-surface temperature has been shown to predict synchronous spawning in corals ${ }^{27}$. The spawning occurrence in the present study coincides with the initial summer-time rise in temperature, which falls in February-March (Figure 11), and not during the peak in May, suggesting that the spawning is triggered by initial warming of seawater. Nonetheless, all the spawning events occurring at night from 2100 to $2130 \mathrm{~h}$ indicate the influence of sunset time and dial cycles, as seen in previous studies over lunar periodicity and tidal influence $^{1,2,6}$.

Scleractinian corals have been observed to spawn synchronously in all parts of the world's reefs ${ }^{2,6}$, which 
Table 2. Rate of survival (number of larvae survived at time t/total population)*100) estimated for two species of Acropora

\begin{tabular}{lccccccccccc}
\hline & \multicolumn{10}{c}{ Days of culture } \\
\cline { 2 - 16 } Species & 6 & 8 & 10 & 12 & 14 & 16 & 18 & 20 & 22 & 24 & 26 \\
\hline A. muricata & $93 \%$ & $87 \%$ & $80 \%$ & $24 \%$ & $24 \%$ & $26 \%$ & $15 \%$ & $9 \%$ & $6 \%$ & $0 \%$ & $0 \%$ \\
A. cytherea & $89 \%$ & $70 \%$ & $75 \%$ & $56 \%$ & $28 \%$ & $21 \%$ & $11 \%$ & $9 \%$ & $10 \%$ & $4 \%$ & $0 \%$ \\
\hline
\end{tabular}

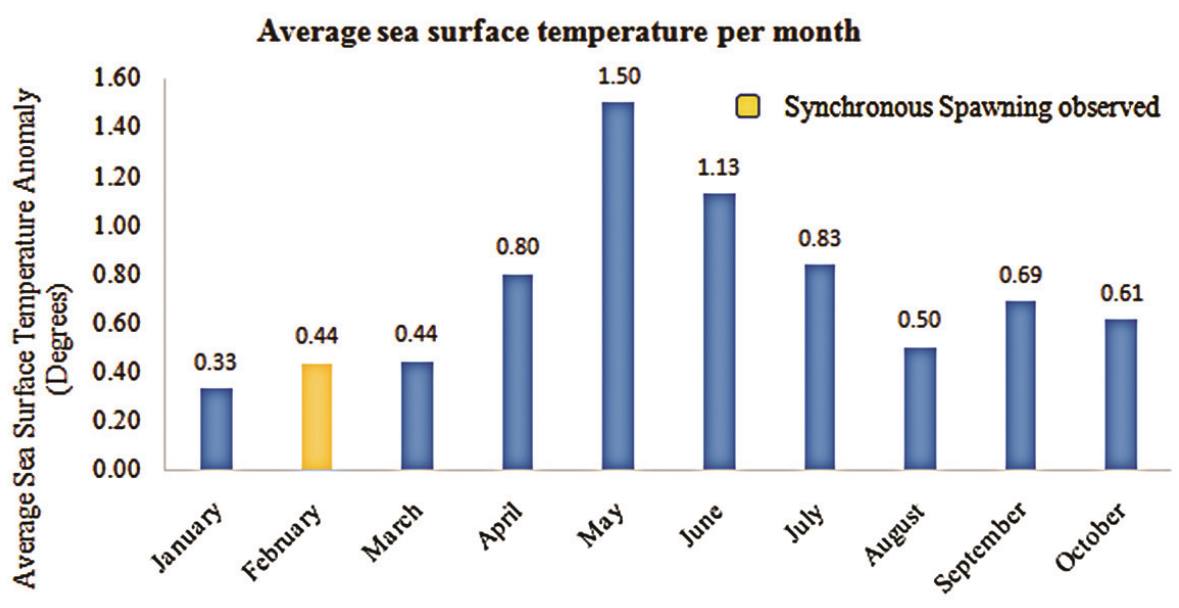

Months of observation in 2018

Figure 11. Sea-surface temperature anomaly for 2018 (ref. 29). The spawning occurrence coincides with the initial summer-time rise in temperature, which falls in February-March.

increases the probability of genetic mixing through crossfertilization and reduces overall predation ${ }^{3,4}$. Multispecific synchronous spawning has been reported from Acropora species in the Indo-Pacific ${ }^{6}$. Our observations confirm synchronous spawning between colonies of Acropora sp. in Lakshadweep, thus confirming the possibility for genetic mixing between colonies, and hence the longterm survival and adaptability of this species in these reefs.

Earlier studies on larval survival and settlement show the larvae of $A$. gemmifera, A. millepora and $A$ valida surviving for a maximum duration of 60, 110, 130 days respectively ${ }^{5}$ - a longer survival duration compared to our estimates. However, Baird ${ }^{5}$ observed that these species could face limited dispersal due to low percentage of survival and limited settlement times. Studies have also reported that the larvae that show peak settlement between 7 and 8 days post-fertilization had lower chances of long-distance dispersal ${ }^{28}$. Our study showed maximum survival duration of 25 and 27 days respectively, for the larvae of $A$. cytheria and A. muricata (Figure 8). Further, we observed a steep die-off beginning from day 12 of the culture, where the rate of survival on day 16 dropped to $21.4 \%$ and $26.0 \%$ for $A$. cytherea and $A$. muricata respectively (Table 2; Figure 8). This indicates that few larvae may be available for settlement during the peak settlement time, i.e. between day 16 and 18 of culture (Figure 9). It is clear that the larvae of Acropora in the present study had comparatively low survival duration than that observed by Baird ${ }^{5}$. The larvae also exhibited low survival rates. These factors could heavily compromise maximum settlement ${ }^{28}$. However, the study reveals that the late onset of settlement time, i.e. from day 12 postspawning and the relatively broader settlement window of 8-10 days (between 12 and 20 days for A. muricata, between 12 and 22 days for $A$. cytherea) could allow room for long-distance dispersal of larvae. Further studies are necessary in this regard.

1. Harrison, P. L., Babcock, R. C., Bull, G. D., Oliver, J. K., Wallace, C. C. and Willis, B. L., Mass spawning in tropical reef corals. Science, 1984, 223(4641), 1186-1189.

2. Babcock, R. C., Bull, G. D., Harrison, P. L., Heyward, A. J., Oliver, J. K., Wallace, C. C. and Willis, B. L., Synchronous spawnings of 105 scleractinian coral species on the Great Barrier Reef. Mar. Biol., 1986, 90(3), 379-394.

3. Guest, J. R., Baird, A. H., Clifton, K. E. and Heyward, A. J., From molecules to moonbeams: spawning synchrony in coral reef organisms. Invertebr. Reprod. Dev., 2008, 51(3), 145-149.

4. Oliver, J. K., Babcock, R. C., Harrison, P. L. and Willis, B. L., Geographic extent of mass coral spawning: clues to ultimate causal factors. In Proceedings of the Sixth International Coral Reef Symposium, Townsville, Australia, 8-12 August 1988.

5. Baird, A. H., The ecology of coral larvae: settlement patterns, habitat selection and the length of the larval phase. Ph D dissertation, James Cook University, Townsville, Australia, 2001, pp. $107-153$.

6. Harrison, P. L. and Wallace, C. C., Reproduction, dispersal and recruitment of scleractinian corals. In Ecosystems of the World: 


\section{RESEARCH ARTICLES}

Coral Reefs (ed. Dubinsky, Z.), Elsevier, Amsterdam, 1990, pp. 133-207.

7. Willis, B. L., Babcock, R. C., Harrison, P. L., Oliver, J. K. and Wallace, C. C., Patterns in the mass spawning of corals on the Great Barrier Reef from 1981 to 1984. In Proceedings of the Fifth International Coral Reef Congress, Tahiti, French Polynesia, 1985.

8. Howells, E. J., Abrego, D., Vaughan, G. O. and Burt, J. A., Coral spawning in the Gulf of Oman and relationship to latitudinal variation in spawning season in the northwest Indian Ocean. Sci. Rep., 2014, 4(1), 1-6.

9. Harrison, P. L., Sexual reproduction of scleractinian corals. In Coral Reefs: An Ecosystem in Transition, Part 3 (eds Dubinsky, Z. and Stambler, N.), Springer, Berlin, Germany, 2011, pp. 59-85.

10. Baird, A. H., Marshall, P. A. and Wolstenholme, J., Latitudinal variation in the reproduction of Acropora in the Coral Sea. In Proceedings of the 9th International Coral Reef Symposium, Bali, Indonesia, 2002.

11. Gilmour, J., Speed, C. W. and Babcock, R., Coral reproduction in Western Australia. PeerJ, 2016, 4, e2010.

12. Mendes, J. M. and Woodley, J. D., Timing of reproduction in Montastraea annularis: relationship to environmental variables. Mar. Ecol. Prog. Ser., 2002, 227, 241-251.

13. SAC, Coral Reef Atlas of the World, Vol. I. Central Indian Ocean, Ahmedabad, 2010, pp. 63-63.

14. Guest, J., Heyward, A., Omori, M., Iwao, K., Morse, A. and Boch, C., Rearing coral larvae for reef rehabilitation. In Reef Rehabilitation Manual (ed. Edwards, A. J.), Coral Reef Targeted Research and Capacity Building for Management Program, St Lucia, Australia, 2010, pp. 73-98

15. Rare phenomenon on the sea. The Hindu, New Delhi, 27 March 2013; https://www.thehindu.com/sci-tech/energy-and-environment/a-rare-phenomenon-on-the-sea/article4551858.ece (accessed on 23 October 2020).

16. Hayashibara, T., Ohike, S. and Kakinuma, Y., Embryonic and larval development and planula metamorphosis of four gametespawning Acropora (Anthozoa, Scleractinia). In Proceedings of the 8th International Coral Reef Symposium, Panama, 1997.

17. Wallace, C. C., Reproduction, recruitment and fragmentation in nine sympatric species of the coral genus Acropora. Mar. Biol. 1985, 88(3), 217-233.

18. Richmond, R. H., Reproduction and recruitment in corals: critical links in the persistence of reefs. In Life and Death of Coral Reefs (ed. Birkeland, C.), Chapman and Hall Publishers, New York, USA, 1997, pp. 175-197.

19. Heyward, A. J. and Negri, A. P., Natural inducers for coral larval metamorphosis. Coral Reefs, 1999, 18(3), 273-279.
20. Harrington, L., Fabricius, K., De'Ath, G. and Negri, A., Recognition and selection of settlement substrata determine postsettlement survival in corals. Ecology, 2004, 85, 3428-3437.

21. Tebben, J. et al., Chemical mediation of coral larval settlement by crustose coralline algae. Sci. Rep., 2015, 5(1), 1-1.

22. Pollock, F. J. et al., Coral larvae for restoration and research: a large-scale method for rearing Acropora millepora larvae, inducing settlement, and establishing symbiosis. PeerJ, 2017, 5, e3732.

23. Raj, K. D. and Edward, J. K. P., Observations on the reproduction of Acropora corals along the Tuticorin coast of the Gulf of Mannar, southeastern India. Indian J. Geomar. Sci., 2010, 39(2), 219-226.

24. Mustafa, A. M. and Silva, M. C. D., Sex and spawning of corals at Port Blair. Islands on March, 1991, 3, 69-70.

25. Boch, C. A., Ananthasubramaniam, B., Sweeney, A. M., Doyle, F. J. and Morse, D. E., Effects of light dynamics on coral spawning synchrony. Biol. Bull., 2011, 220(3), 161-173.

26. Jokiel, P. L., Ito, R. Y. and Liu, P. M., Night irradiance and synchronization of lunar release of planula larvae in the reef coral Pocillopora damicornis. Mar. Biol., 1985, 88(2), 167-174.

27. Hayashibara, T. et al., Patterns of coral spawning at Akajima Island Okinawa, Japan. Mar. Ecol. Prog. Ser., 1993, 25, 253-262.

28. Nozawa, Y. and Harrison, P. L., Effects of elevated temperature on larval settlement and post-settlement survival in scleractinian corals, Acropora solitaryensis and Favites chinensis. Mar. Biol., 2007, 152(5), 1181-1185.

29. https://www.ospo.noaa.gov/data/sst/anomaly/2018 (accessed on 8 December 2018).

ACKNOWLEDGEMENTS. We acknowledge funding support from the Department of Environment and Forests, Administration of the Union Territory (UT) of Lakshadweep, under the project 'Conservation management of coral reefs in Lakshadweep-long-term monitoring programme'. We thank Dr Kailash Chandra, Director, Zoological Survey of India and Dr K. Venkataraman (former Director, Zoological Survey of India), for approval to undertake this study and continuous encouragement; and Shri Damodhar Naik, Conservator of Forests, UT of Lakshadweep, for permission to undertake this study, providing the necessary logistics of laboratory space, and support during the study. The monitoring, assistance in planning and logistics during the study by the Nodal Officers and Monitoring staff of the project is acknowledged. We also thank the two anonymous reviewers for useful comments that helped improve the manuscript.

Received 27 October 2020; revised accepted 7 April 2021

doi: $10.18520 / \mathrm{cs} / \mathrm{v} 121 / \mathrm{i} 1 / 133-140$ 